\title{
Are the maximum hardness and minimum polarizability principles always obeyed in nontotally symmetric vibrations?
}

\author{
Miquel Torrent-Sucarrat, Josep M. Luis, Miquel Duran, and Miquel Solà \\ Institut de Química Computacional and Departament de Química, Universitat de Girona, \\ E-17071 Girona, Catalonia, Spain
}

(Received 7 February 2002; accepted 9 September 2002)

\begin{abstract}
In a recent paper [J. Am. Chem. Soc. 123, 7951 (2001)] we have shown for the first time the existence of molecules with nontotally symmetric vibrational modes that break the maximum hardness (MHP) and minimum polarizability (MPP) principles. We present here an extension of this previous work by devising a mathematical procedure that helps to determine the nontotally symmetric molecular distortions of a given molecule that do not follow the MPP or the MHP. This methodology is based on the diagonalization of the Hessian matrix of the polarizability or the hardness with respect to the vibrational normal coordinates. For a relatively large series of molecules, we have carried out diagonalizations of the Hessian matrix of the polarizability to determine the molecular distortions with a more marked MPP or anti-MPP character. From the results obtained, we have derived a set of simple rules that allow to predict a priori without calculations the existence of vibrational modes that break the MPP. With respect to the MHP, the results strongly depend on the method of calculation, but the same rules are useful to predict the existence of vibrational modes that disobey the MHP when the Koopmans' approximation is used to calculate the hardness. ㄷ 2002 American Institute of Physics. [DOI: 10.1063/1.1517990]
\end{abstract}

\section{INTRODUCTION}

The quest for principles, laws, theorems, postulates, or rules that rationalize the large body of available scattered information on chemical reactions is a major task of chemists. ${ }^{1}$ The most traditional chemical reactivity principles are those based on thermodynamics, the maximum entropy principle ${ }^{2}$ being the most significant example of this group. The change in molecular structure when going from reactants to products through the transition state is the key aspect of a second branch of reactivity principles. Among these principles founded on molecular structure, the most important is probably the Hammond's postulate formulated in $1955 .^{3}$ Finally, there is a set of electronic structure reactivity principles that monitor the evolution along the reaction coordinate of some electronic properties, such as the electronegativity, hardness, polarizability, bond order, frontier orbitals, etc. In this last group one can make a distinction between the principles based on general quantum theory, for instance, the Hückel rule, ${ }^{4}$ the Woodward-Hoffmann rules, ${ }^{5}$ and the maximum molecular valence principle, ${ }^{6}$ and those that are rooted in the density functional theory (DFT), such as the Sanderson electronegativity equalization principle (EEP), ${ }^{7}$ the hard-soft acid-base principle (HSAB), ${ }^{8,9}$ the maximum hardness principle (MHP), ${ }^{9-11}$ and minimum polarizability principle (MPP). ${ }^{12,13}$

Actually, the MHP and MPP principles are among the most widely accepted electronic structure principles of chemical reactivity. The MHP affirms that, at a given temperature, molecular systems evolve to a state of maximum hardness. The MPP was formulated on the basis of the MHP and an inverse relationship between hardness and polarizability. ${ }^{14}$ This principle states that the natural direction of evolution of any system is towards a state of minimum polarizability. Both principles have been applied successfully to the study of molecular vibrations, ${ }^{9,15-18}$ internal rotations, ${ }^{6(b), 19}$ excited states, ${ }^{20,21}$ aromaticity, ${ }^{22}$ and different types of chemical reactions. ${ }^{12,23-33}$ It has been found in most of these cases that the conditions of maximum hardness and minimum polarizability complement the minimum energy criterion for molecular stability.

A formal proof of the MHP based on statistical mechanics and the fluctuation-dissipation theorem was given by Parr and Chattaraj ${ }^{11}$ under the constraints that the chemical potential and the so-called external potential $(v(\mathbf{r}))$ must remain constant upon distortion of molecular structure. Here $v(\mathbf{r})$ is the potential acting on an electron at $\mathbf{r}$ due to the nuclear attraction plus such other external forces as may be present. These are two severe constraints that are usually not fulfilled. However, relaxation of these constraints seems to be permissible, and in particular, it has been found that in most cases the MHP still holds even though the chemical and external potentials vary during the molecular vibration, internal rotation or along the reaction coordinate. ${ }^{12,15-33}$ Hereafter, we will refer to the generalized MHP (GMHP) or MPP (GMPP) as the maximum hardness or minimum polarizability principles that do not require the constancy of chemical and external potentials during molecular change. It is worth emphasizing that the generalized versions of these principles have not been proven.

Given the inherent complexity of chemical reactions it is difficult to find principles of chemical reactivity that can be applied to any reaction. In fact, apart from the principles based on thermodynamics, most principles of chemical reactivity are qualitative and they do not have a general applica- 
bility. For instance, this is the case of the Hammond postulate for which a number of failures have been reported. $^{29,34-36}$ It is also the situation of the GMHP and GMPP that fail in some chemical reactions ${ }^{26,29,33}$ and excited states. $^{21}$ In most of these cases it has been found that the chemical and external potentials change noticeably during the process. Therefore these observations do not violate the strict MHP because this principle is rigorously valid only under constant chemical and external potentials.

In addition to chemical reactions, the molecular motion along a nontotally symmetric vibration is also an interesting case in point to analyze from the viewpoint of the MHP and MPP. Let us start with a molecule in its equilibrium geometry and make a displacement from equilibrium along a nontotally symmetric normal mode. For this particular molecular motion and using symmetry arguments, Pearson and Palke ${ }^{15}$ showed that the values of the average external potential $\left(v_{e n}\right)$, hardness $(\eta)$, polarizability $(\alpha)$, and chemical potential $(\mu)$ for the positive deviation will be the same as those for the negative deviation from equilibrium. Then, if $Q$ represents a nontotally symmetric normal mode coordinate, it follows that $(\delta \eta / \delta Q)=(\delta \alpha / \delta Q)=0$, and more important, $(\delta \mu / \delta Q)=\left(\delta v_{e n} / \delta \mathrm{Q}\right)=0$ at the equilibrium geometry, $v_{e n}$ being the electron-nuclear attraction potential energy. After a small displacement, $\Delta Q$, from the equilibrium geometry, the average external potential may be written as

$$
v_{\mathrm{en}}=v_{\mathrm{en}}^{o}+\left(\frac{\delta v_{\mathrm{en}}}{\delta Q}\right) \Delta Q+\cdots,
$$

and, therefore, since $\left(\delta v_{\mathrm{en}} / \delta Q\right)=0$ we have that for small nontotally symmetric displacements $v_{\text {en }}$ is approximately constant. The same applies to the chemical potential. Hence, the chemical and external potential are roughly constant ${ }^{37}$ for small distortions along nontotally symmetric normal modes, thus nearly following the two conditions of Parr and Chattaraj. ${ }^{11}$ As a consequence, the MHP and MPP are expected to be obeyed for nontotally symmetric vibrations, as confirmed by most numerical calculations of hardness and polarizability along the nontotally symmetric normal modes performed so far. ${ }^{9,15-18}$ For totally symmetric distorsions, the situation is drastically different. Now, starting from the equilibrium geometry the hardness keeps increasing steadily as the nuclei approach each other. Therefore, the equilibrium structure is not a maximum of hardness for displacements along totally symmetric normal modes. This is not a violation of the strict MHP since neither the chemical nor the external potentials are kept constant during this kind of distortion.

We have recently shown ${ }^{38}$ for the first time the existence of nontotally symmetric vibrational modes that break the generalized versions of the MHP and MPP. We have found that the modes that disobey the GMHP and GMPP have the characteristic nuclear displacements of bond length alternation (BLA) modes. In contrast to the examples of a breakdown of the GMHP and GMPP reported to date, ${ }^{21,26,29,33}$ this is an example of a failure of these principles for the most favorable case in which the external and chemical potentials keep nearly constant. Remarkably, we have found that molecules such as pyridine, benzene, naphthalene, or byphe- nylene possess nontotally symmetric BLA modes that violate the GMHP and GMPP, whereas others such as furan, cyclopentadiene, cyclooctatetraene, or benzocyclobutadiene do not.

The aim of the present work is to report the results obtained for these systems (only pyridine was described in the previous work ${ }^{38}$ ) and to explain the reasons for the different behavior among these related molecules.

\section{COMPUTATIONAL DETAILS}

All geometry optimizations and chemical potential, hardness, polarizability, and frequency calculations have been performed at the Hartree-Fock (HF), hybrid density functional B3LYP, ${ }^{39}$ second-order Møller-Plesset (MP2), ${ }^{40}$ and singles and doubles quadratic configuration interaction (QCISD) (Ref. 41) methods using Pople standard basis sets, ${ }^{42}$ such as the $6-31 \mathrm{G}$ and the $6-311 \mathrm{G}^{* *}$ basis sets, and also the aug-cc-pVTZ (Ref. 43) basis set. All calculations have been carried out with the GAUSSIAN 98 package. ${ }^{44}$

The exact definitions of $\mu$ and $\eta$ were given by Parr and Pearson $^{9,45}$ in the framework of the conceptual density functional theory as

$$
\begin{aligned}
& \mu=\left(\frac{\partial E}{\partial N}\right)_{\nu(\mathbf{r})}, \\
& \eta=\left(\frac{\partial^{2} E}{\partial N^{2}}\right)_{\nu(\mathbf{r})}=\left(\frac{\partial \mu}{\partial N}\right)_{\nu(\mathbf{r})} .
\end{aligned}
$$

A three-points finite difference approximation leads to the following working definitions of these quantities,

$$
\begin{aligned}
& \mu=-\frac{1}{2}(I+A), \\
& \eta=I-A .
\end{aligned}
$$

$I$ and $A$ are the first vertical ionization potential and electron affinity of the neutral molecule, respectively. For the calculation of $I$ and $A$, the energy of the cationic and anionic doublet species has been computed within the unrestricted methodology at the geometry of the neutral systems, while the neutral singlet molecules have been calculated using the restricted formalism.

The Koopmans' theorem $\left(I \approx-\varepsilon_{H}\right.$ and $\left.A \approx-\varepsilon_{L}\right)$ allows one to write $\mu$ and $\eta$ in terms of the energy of frontier HOMO $\left(\varepsilon_{H}\right)$ and LUMO $\left(\varepsilon_{L}\right)$ molecular orbitals of the reference species. ${ }^{9,45}$ For closed shell species one obtains, ${ }^{46}$

$$
\begin{aligned}
& \mu=\frac{1}{2}\left(\varepsilon_{L}+\varepsilon_{H}\right), \\
& \eta=\varepsilon_{L}-\varepsilon_{H} .
\end{aligned}
$$

It is worth noting that given the particular definition of $\mu$ and $\eta$ [Eqs. (2) and (3)], 9,45 all operational equations that provide $\mu$ and $\eta$ values, and, in particular Eqs. (4)-(7), are approximate. $^{48}$

Finally, the isotropic average polarizability has been obtained using

$$
\alpha=\frac{\alpha_{x x}+\alpha_{y y}+\alpha_{z z}}{3} .
$$


TABLE I. HF, B3LYP, MP2, and QCISD static isotropic average polarizabilities, chemical potentials, and hardness for the molecular distortions of benzene along the two $B_{2 U}$ normal vibrational modes depicted in Fig. 1. ${ }^{\mathrm{a}}$

\begin{tabular}{|c|c|c|c|c|c|c|c|c|c|c|c|}
\hline \multirow[b]{2}{*}{ Property } & & \multicolumn{3}{|c|}{$\mathrm{HF}$} & \multicolumn{3}{|c|}{ B3LYP } & \multicolumn{2}{|c|}{ MP2 } & \multicolumn{2}{|c|}{ QCISD } \\
\hline & & $6-31 \mathrm{G}$ & $6-311 \mathrm{G}^{* *}$ & aug-cc-pVTZ & $6-31 \mathrm{G}$ & $6-311 G^{* *}$ & aug-cc-pVTZ & $6-31 \mathrm{G}$ & $6-311 G^{* *}$ & $6-31 \mathrm{G}$ & $6-311 \mathrm{G}^{* *}$ \\
\hline \multirow[t]{3}{*}{$\alpha$} & Eq. & 51.09808 & 56.79026 & 66.74075 & 53.33033 & 59.17784 & 69.33744 & 52.98986 & 58.24366 & 51.36533 & 57.33009 \\
\hline & $1 B_{2 U} \pm 0.04^{\mathrm{b}}$ & 51.09581 & 56.78353 & 66.73066 & 53.33136 & 59.17883 & 69.33821 & 52.99057 & 58.24501 & 51.36415 & 57.32976 \\
\hline & $2 B_{2 U} \pm 0.04^{\mathrm{b}}$ & 51.09690 & 56.79069 & 66.74038 & 53.31558 & 59.16757 & 69.32336 & 52.96688 & 58.21445 & 51.36338 & 57.32384 \\
\hline \multirow[t]{3}{*}{$-2 \mu_{1}$} & Eq. & 0.185837 & 0.199504 & 0.308492 & 0.244452 & 0.265797 & 0.278068 & 0.188612 & 0.201079 & 0.188872 & 0.201181 \\
\hline & $1 B_{2 U} \pm 0.04^{\mathrm{b}}$ & 0.185841 & 0.199507 & 0.308536 & 0.244457 & 0.265801 & 0.278072 & 0.188617 & 0.201084 & 0.188875 & 0.201186 \\
\hline & $2 B_{2 U} \pm 0.04^{\mathrm{b}}$ & 0.185847 & 0.199513 & 0.308509 & 0.244430 & 0.265783 & 0.278059 & 0.188621 & 0.201093 & 0.188881 & 0.201193 \\
\hline \multirow[t]{3}{*}{$\eta_{1}$} & Eq. & 0.482066 & 0.472429 & 0.368479 & 0.251955 & 0.246958 & 0.242999 & 0.471510 & 0.466611 & 0.471236 & 0.466300 \\
\hline & $1 B_{2 U} \pm 0.04^{\mathrm{b}}$ & 0.482105 & 0.472513 & 0.368523 & 0.251957 & 0.246962 & 0.243003 & 0.471515 & 0.466614 & 0.471262 & 0.466318 \\
\hline & $2 B_{2 U} \pm 0.04^{\mathrm{b}}$ & 0.482120 & 0.472457 & 0.368495 & 0.252137 & 0.247088 & 0.243114 & 0.471828 & 0.467079 & 0.471312 & 0.466437 \\
\hline \multirow[t]{3}{*}{$-2 \mu_{2}$} & Eq. & 0.343657 & 0.357857 & 0.373143 & 0.248669 & 0.270304 & 0.325148 & 0.296006 & 0.338065 & 0.325000 & 0.367113 \\
\hline & $1 B_{2 U} \pm 0.04^{\mathrm{b}}$ & 0.345388 & 0.359288 & 0.373036 & 0.248423 & 0.270394 & 0.325153 & 0.299484 & 0.338113 & 0.324980 & 0.367378 \\
\hline & $2 B_{2 U} \pm 0.04^{\mathrm{b}}$ & 0.343491 & 0.359347 & 0.373015 & 0.248551 & 0.270299 & 0.325285 & 0.314677 & 0.335354 & 0.324858 & 0.366865 \\
\hline \multirow[t]{3}{*}{$\eta_{2}$} & Eq. & 0.576654 & 0.566325 & 0.549814 & 0.418974 & 0.408187 & 0.356377 & 0.532549 & 0.513037 & 0.546491 & 0.532828 \\
\hline & $1 B_{2 U} \pm 0.04^{\mathrm{b}}$ & 0.574521 & 0.564908 & 0.549767 & 0.419015 & 0.408275 & 0.356380 & 0.529137 & 0.513088 & 0.546489 & 0.532546 \\
\hline & $2 B_{2 U} \pm 0.04^{\mathrm{b}}$ & 0.576528 & 0.564908 & 0.549695 & 0.419066 & 0.408325 & 0.356515 & 0.544568 & 0.515787 & 0.546403 & 0.532673 \\
\hline
\end{tabular}

${ }^{\mathrm{a}} \alpha, \mu_{1}, \eta_{1}, \mu_{2}$, and $\eta_{2}$ are calculated using Eqs. (8), (6), (7), (4), and (5), respectively, all these values are given in a.u. $\mu_{1}$ and $\eta_{1}$ are calculated using the HOMO and LUMO energies (in hartrees) of the Hartree-Fock molecular orbitals, except for the B3LYP method for which the Kohn-Sham orbital energies were used.

${ }^{\mathrm{b}}$ The distortions are given in bohrs.

It is important to remark that polarizabilities and frequencies in the GAUSSIAN 98 package are calculated analytically at the HF, B3LYP, and MP2 levels of theory and numerically when the QCISD method is used. The Hessian of the polarizability with respect to the nontotally symmetric normal coordinates (vide infra) has been calculated by numerical differentiation of the analytical first derivative of the polarizability with respect to the normal coordinates.

\section{RESULTS AND DISCUSSION}

We shall begin our discussion by considering two nontotally symmetric modes of benzene that disobey the GMHP and GMPP. We will show that the results are basis set and method dependent. After that, a method for automatic detection of nontotally symmetric motions that break the GMPP or the GMHP will be presented, although it will be applied for the GMPP only. The results obtained with this method experience no dependence on the basis set and method at least for all the studied systems as far as the GMPP is concerned. Finally, we will derive a set of simple rules to a priori predict without calculations whether a given molecule will possess nontotally symmetric modes that disobey the GMPP. The usefulness of these rules to determine the existence of vibrational modes that break the GMHP will be also discussed.

\section{A. The benzene molecule as a test example}

Table I collects the HF, B3LYP, MP2, and QCISD values of the hardness [Eqs. (5) and (7)], chemical potential [Eqs. (4) and (6)], and polarizability corresponding to the equilibrium structure of benzene and to structures obtained from positive and negative displacements ( \pm 0.04 bohrs) along the two $B_{2 U}$ modes depicted in Fig. 1 for the different basis sets analyzed. In this figure it is clearly seen that the $B_{2 U}$ vibrational modes have the characteristic nuclear displacements of
BLA modes. The values of polarizability at the HF/aug-ccpVTZ level in Table I show that for these two modes the equilibrium structure is a maximum of polarizability in contradiction with the GMPP. The increase in the polarizability along these two $B_{2 U}$ modes can be attributed to a certain localization of the $\pi$ electrons during the BLA vibration or alternatively to an increase of the HOMO-LUMO gap (vide infra) in a similar way to what we have observed for pyridine. ${ }^{38}$ The results obtained at the HF level using the aug-cc-pVTZ basis set are also found with the 6-31G basis set, while for the $6-311 \mathrm{G}^{* *}$ basis set only the $1 B_{2 U}$ mode violates the GMPP.

With respect to the hardness, the HF results are more dependent on the scheme used for the calculation [Eqs. (5) or (7)] than on the basis set used. For all basis sets, the two $B_{2 U}$ vibrational modes of Fig. 1 break the GMHP when the hardness is computed using Eq. (7), while the same modes obey the GMHP according to the HF values of the hardness computed with Eq. (5). Thus, for this system, we observe diametrically opposed trends when the $\eta$ values are computed using Eqs. (5) or (7). This is remarkable because it is a common belief that, although the numerical values may differ, the overall qualitative trends remain unaltered when $\eta$ is calculated using Eqs. (5) or (7). ${ }^{19,29}$ For a given nontotally symmetric nuclear distortion, hardnesses obtained from Eq. (7) tend to disobey more often the GMHP than those calculated from Eq. (5) (vide infra). In particular, we have found several vibrational modes, apart from the two $B_{2 U}$ modes depicted in Fig. 1, that disobey the GMHP when the hardness is calculated through Eq. (7). ${ }^{47}$ However, the largest deviations from the GMHP occurs in the two $B_{2 U}$ modes studied.

The increase in the hardness value obtained using Eq. (7) after a small displacement along the two $B_{2 U}$ modes studied is mainly due to a stabilization of the degenerate HOMO in benzene. The LUMO energy remains either approximately 


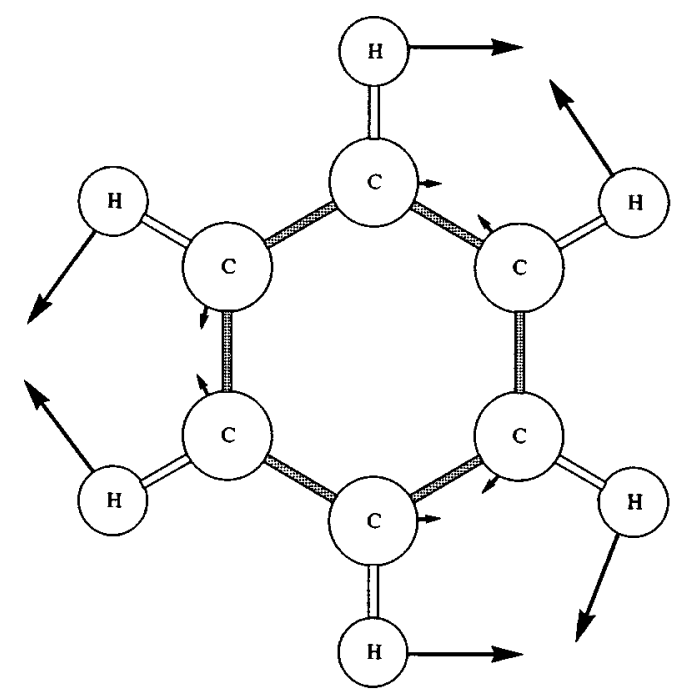

$\mathbf{1 B}_{\mathbf{2 U}}$

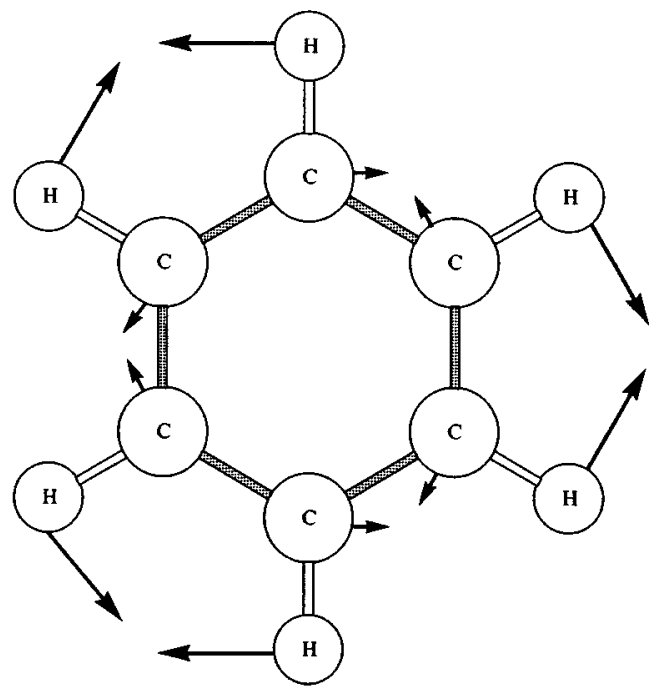

$\mathbf{2 B}_{\mathbf{2 U}}$

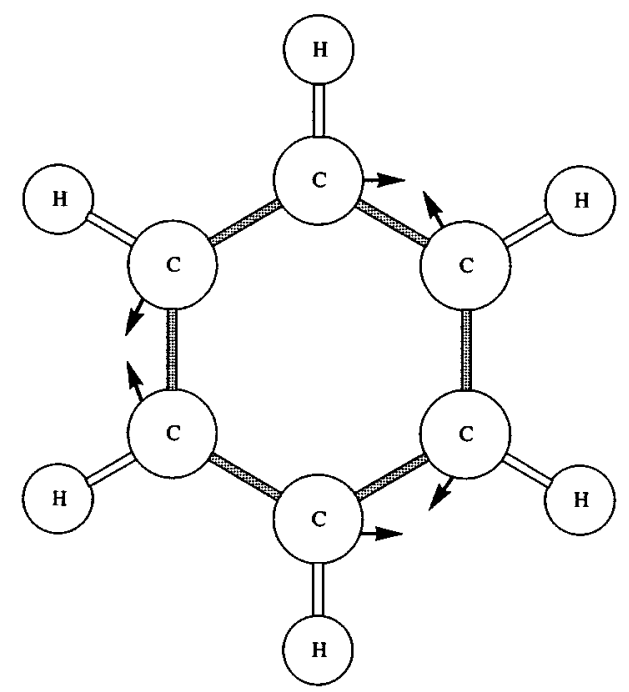

$\mathbf{1 B}_{\mathbf{2}} \mathbf{U}^{\prime}$

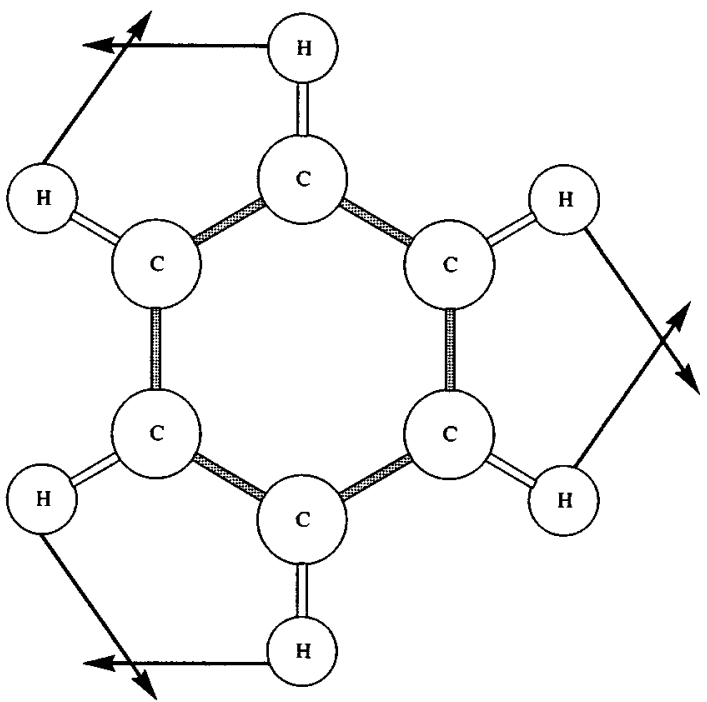

$\mathbf{2 B}_{\mathbf{2}} \mathbf{U}^{\prime}$

FIG. 1. Schematic representation of the displacement vectors corresponding to the two $B_{2 U}$ studied normal vibrational modes of benzene ( $1 B_{2 U}$ and $2 B_{2 U}$ ) and the two $B_{2 U}$ postdiagonalitzation nuclear distortions of benzene $\left(1 B_{2 U^{\prime}}\right.$ and $\left.2 B_{2 U^{\prime}}\right)$. The displacement vectors have been calculated at the HF/6-31G level.

constant or increases with the $B_{2 U}$ vibrations. The stabilization of the HOMO can be understood by observing the shape of this degenerate orbital [Fig. 2(a)] and the BLA distortion of the $B_{2 U}$ vibrational modes (Fig. 1). The decrease in the HOMO energy is basically produced by the interaction between adjacent $\mathrm{C}$ atoms as the molecule vibrates. The increase in the bonding interaction when two adjacent $\mathrm{C}$ atoms approach is more important than the reduction of the same bonding interaction as the $\mathrm{C}$ atoms move away. As a result the HOMO is stabilized and the hardness increases in contradiction with the GMHP.

One could attribute the breakdown of the GMHP and GMPP in these two $B_{2 U}$ normal modes to the fact that the chemical potential and the external potential change significantly during these vibrations. However, this is not the case since we have found that variations in chemical and external potentials are similar among all nontotally symmetric modes. In fact, at the HF/6-311G** level, the $1 B_{2 U}$ mode that disobeys the GMPP presents a variation of chemical potential upon distortion smaller than the $2 B_{2 U}$ mode that follows the GMPP (see Table I). Finally, we have checked that the HF results derived from Table I using displacements of \pm 0.04 bohrs remain qualitatively unaltered when using displacements of $\pm 0.02, \pm 0.08$, and \pm 0.16 bohrs.

The behavior of these two $B_{2 U}$ modes with respect to the GMPP and GMHP is not only basis set dependent, but also depends clearly on the methodology used to calculate the wave function or the electron density as one can see in Table I. Thus, concerning the GMPP, at the B3LYP and MP2 levels, the $2 B_{2 U}$ mode always disobeys the GMPP and the 

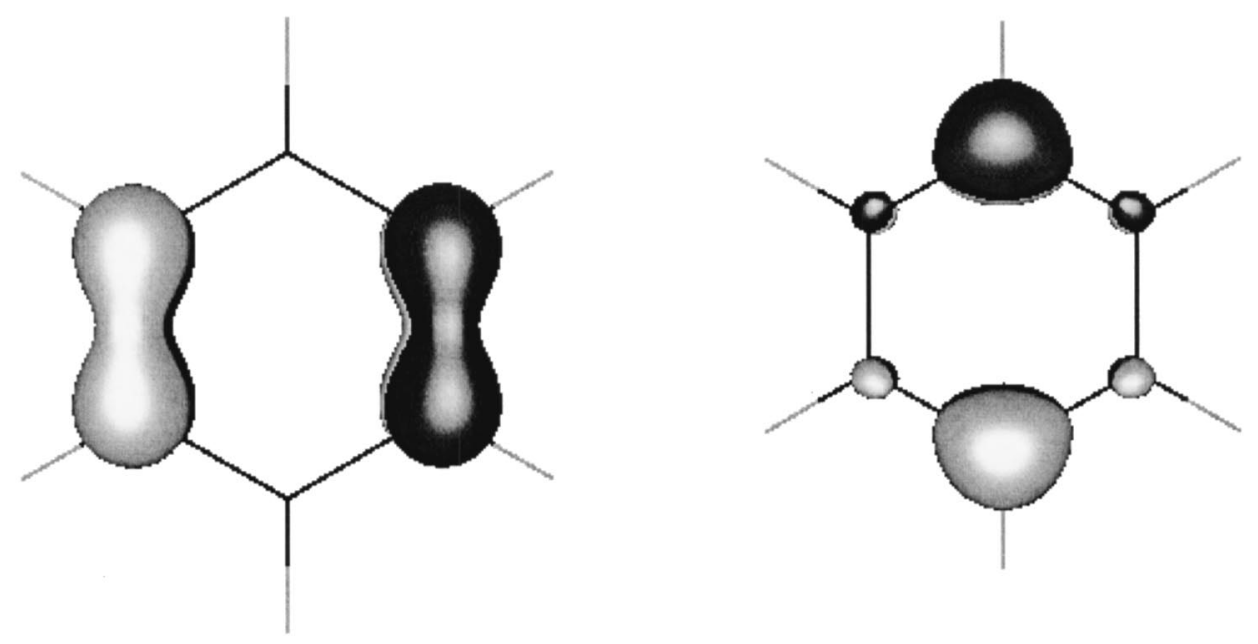

a)

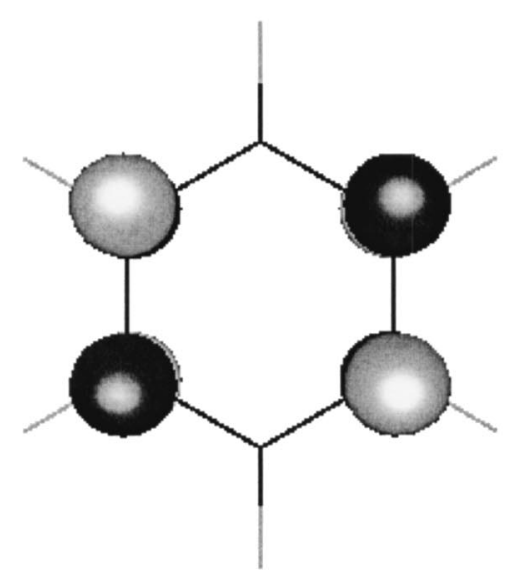

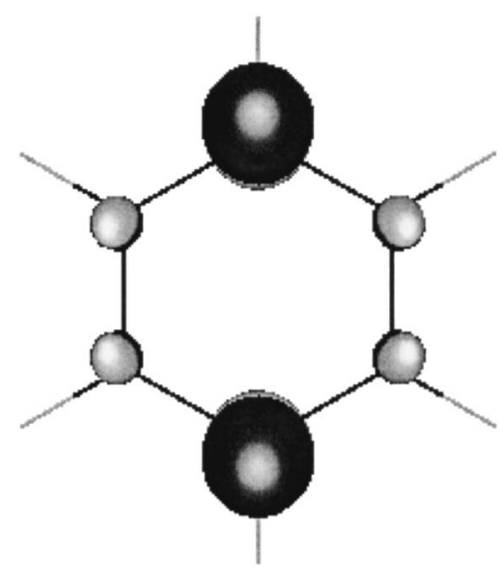

FIG. 2. Representation of the B3LYP/6-311G** isosurfaces 0.1 (black) and -0.1 (grey) a.u. of the (a) degenerate HOMO and (b) degenerate LUMO of benzene.

b)

$1 B_{2 U}$ follows it with all basis sets used. On the other hand, with the QCISD method (Table I) one finds that the two modes violate the GMPP with all basis sets analyzed. With regard to the GMHP, the two modes violate this principle for all methods and basis sets analyzed when using Eq. (7) to calculate the hardness. The same result is obtained from Eq. (5) using the aug-cc-pVTZ, 6-311G**, and 6-31G basis sets in conjunction with the B3LYP method. Finally, Eq. (5) provides almost opposite results for the MP2 and QCISD methods. Thus, according to the QCISD method, all modes obey the GMHP for the two different basis sets studied, while the opposite is found when using the MP2 method, except for the $1 B_{2 U}$ calculated with the MP2/6-31G method.

It is interesting to remark that, in general, for a given level of theory, the modes that violate the GMPP, more manifestly break the GMHP when the hardness is calculated within the Koopmans' approximation [Eq. (7)]. Thus, for instance, at the MP2/6-311G** level, the $2 B_{2 U}$ vibrational mode disobeys the GMPP while the $1 B_{2 U}$ mode conform it. Accordingly, the distortion along the $2 B_{2 U}$ vibrational mode has the largest increase in hardness when compared to the equilibrium value.

In summary, we have demonstrated here that there two
BLA vibrational modes in benzene that may break the GMPP and GMHP. However, the results exhibit a great dependence on the method and basis set used, and in the case of the hardness also on the scheme employed for estimating its value.

\section{B. The diagonalization of the polarizability Hessian matrix}

We have shown in the previous section that some nontotally symmetric modes may have GMPP or anti-GMPP character depending on the methodology used for the calculations. We will discuss now a method to find nontotally symmetric distortions with GMPP or anti-GMPP character independent on the procedure of computation used. This method is based on the diagonalization of the Hessian matrix of the polarizability with respect to the nontotally symmetric normal coordinates, whose elements are obtained as

$$
A_{k l}=\left(\frac{\partial^{2} \alpha}{\partial Q_{k} \partial Q_{l}}\right),
$$

with $k$ and $l$ running over the nontotally symmetric modes. The diagonalization of the Hessian matrix of the polarizabil- 


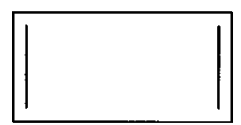

$\mathbf{D}_{2} \mathbf{h}$
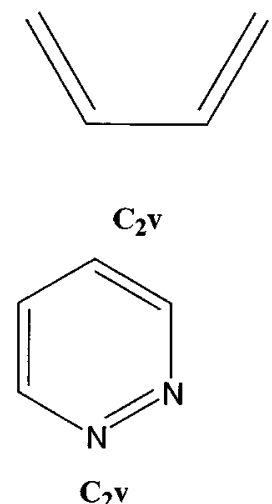

$\mathrm{C}_{2} \mathrm{v}$
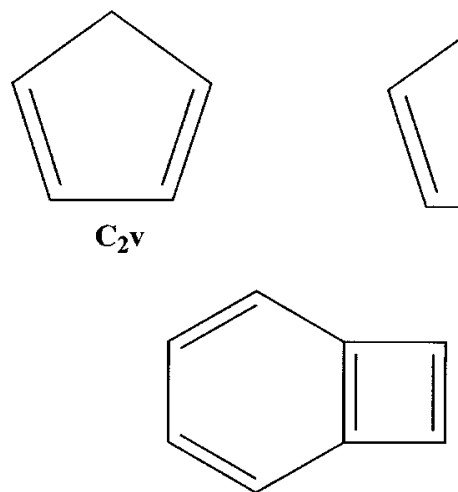

$\mathrm{C}_{2} \mathrm{v}$

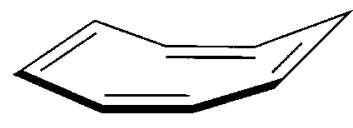

$\mathbf{D}_{2} \mathbf{d}$
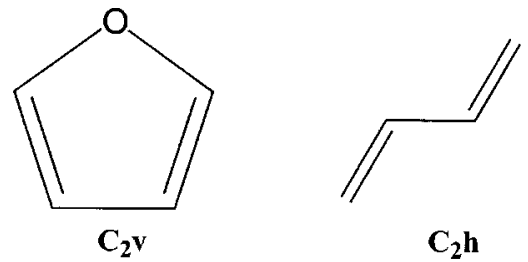

$\mathrm{C}_{2} \mathrm{~h}$

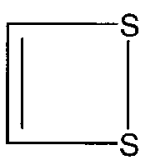

FIG. 3. A series of molecules with nontotally symmetric vibrational modes that satisfy the minimum polarizability principle without exception. ity provides the nontotally symmetric distortions that produce the largest polarizability changes, which correspond to nuclear displacements that have a more marked GMPP or anti-GMPP character than the original vibrational modes. Obviously, this method can be used to determine the nuclear displacements that have a more marked GMHP or antiGMHP character by just changing the polarizability by the hardness in Eq. (9). In this work, we have restricted our study to the polarizability for two reasons. First, the computational effort required to calculate the Hessian matrix of the hardness is larger by several orders of magnitude due to the lack of analytical first and second derivatives of the hardness with respect to the vibrational normal coordinates. Second, whereas the polarizability can be computed exactly at any level of theory, hardness values obtained from different theoretical approaches are all approximate. ${ }^{48}$

Makov $^{17}$ demonstrated from symmetry considerations that molecular properties such as the hardness or the polarizability at the equilibrium geometry are an extremum, which could be either a minimum or a maximum, with respect to distortions along nontotally symmetric normal coordinates. Therefore, the sign of the diagonal terms of matrix A tell us whether a certain nontotally symmetric vibrational mode has GMPP or anti-GMPP character, thus giving us exactly the same information that the calculations performed in the previous section.

On the other hand, the eigenvectors obtained in the diagonalization of the A matrix are linear combinations of nontotally symmetric vibrational modes (for a given eigenvector all implicated vibrational modes belong to the same symmetry species) giving the distortions that produce the largest polarizability changes. In this case, the sign of the eigenvalue characterizes the GMPP or anti-GMPP character of the distortion along a given eigenvector. If an eigenvector has a negative eigenvalue, the equilibrium structure will represent a maximum of polarizability along this distortion, which consequently does not fulfill the GMPP. On the contrary, if the eigenvalue is positive, the equilibrium structure will represent a minimum of polarizability along this distortion, which therefore follows the principle.

Figure 1 depicts the two nontotally symmetric vibrational modes of benzene that according to the previous section may break the GMPP ( $1 B_{2 U}$ and $\left.2 B_{2 U}\right)$ and the two postdiagonalization distortions $\left(1 B_{2 U^{\prime}}\right.$ and $\left.2 B_{2 U^{\prime}}\right)$ computed at the HF/6-31G level. The $1 B_{2 U^{\prime}}$ distortion is a positive linear combination of the original $1 B_{2 U}$ and $2 B_{2 U}$ vibrational modes $\left(0.762 * 1 B_{2 U}+0.648 * 2 B_{2 U}\right)$ having a negative eigenvalue. In the $1 B_{2 U}$, distortion, only the nuclear displacements of the carbon atoms are significant and have a clear BLA character. This fact corroborates one of the conclusions of our previous work, ${ }^{38}$ i.e., the importance of the BLA distortions of the heavier atoms on the breakdown of the GMPP. In contrast, the $2 B_{2 U^{\prime}}$ has a positive eigenvalue and it is a negative linear combination of the original $1 B_{2 U}$ and $2 B_{2 U}$ vibrational modes $\left(-0.648 * 1 B_{2 U}\right.$ $\left.+0.762 * 2 B_{2 U}\right)$. In this $2 B_{2 U^{\prime}}$ distortion, the displacements of the carbon atoms have been reduced and those of the hydrogen atoms have been increased.

We have applied this methodology to 17 molecules grouped in two different sets corresponding to the molecules of Figs. 3 and 4 using the HF/6-31G method. All diagonal terms and eigenvalues of the A matrix of molecules in Fig. 3 are positive; therefore all the pre- and postdiagonalization nontotally symmetric distortions of these molecules are consistent with the GMPP. On the contrary, the systems of the Fig. 4 display one or more negative eigenvalues of the $\mathbf{A}$ matrix (see Table II); consequently these molecules have one or more nontotally symmetric distortions that break the GMPP. In all studied molecules the postdiagonalization distortions with negative eigenvalues are the result of a linear combination of the nontotally symmetric vibrational modes that maximizes the BLA displacement.

The validity of these results have been confirmed in the case of the benzene molecule at the HF level using the 
<smiles>c1ccccc1</smiles><smiles>c1ccncc1</smiles>

$D_{6}$ h 2(1) $C_{2}$ V 1(1)

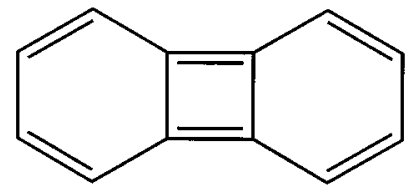

$\mathbf{D}_{2}$ h 3(2)<smiles>c1cnccn1</smiles>

$\mathbf{D}_{2}$ h 2(1)<smiles>c1cncnc1</smiles>

$\mathrm{C}_{2} \mathrm{v}$ 1(1)
FIG. 4. List of molecules that possess one or more nontotally symmetric distortions that break the minimum polarizability principle. Beside the symmetry group of the molecule there are the number of negative eigenvalues and diagonal terms of the A matrix, in brackets and without brackets, respectively.
$D_{4}$ h 4(2)

6-31G, 6-311G**, and aug-cc-pVTZ basis sets and at the B3LYP, MP2, and QCISD methods using the 6-31G basis set (see Table III). Qualitatively, the results are equivalent with all methods and basis sets tested, thus reinforcing the belief that the postdiagonalization results are basically basis set and method independent.

One probably may anticipate that nuclear displacements having a marked anti-GMPP character (the $1 B_{2 U^{\prime}}$ distortion in benzene) have also a clear anti-GMHP behavior. To check this hypothesis, we have calculated the hardness using different methods and basis sets for the molecular displacements of benzene along the two $B_{2 U^{\prime}} \mathrm{HF} / 6-31 \mathrm{G}$ postdiagonalization distortions depicted in Fig. 1 (Table III). As for the original $B_{2 U}$ vibrations, we have found that the results obtained are quite dependent on the scheme used to calculate the hardness. Thus, the two $B_{2 U^{\prime}}$ distortions break the GMHP when using hardnesses values from Eq. (7) irrespective of the level of theory used. On the other hand, the

TABLE II. HF/6-31G diagonal terms and eigenvalues of the Hessian matrix of the polarizability with respect to the nontotally symmetric modes. All values are given in a.u.

\begin{tabular}{llcr}
\hline \hline Molecules & & $A_{k k}$ & Eigenvalues \\
\hline Benzene $\left(D_{6 h}\right)$ & $1 B_{2 U}$ & -0.4253 & -1.0766 \\
& $2 B_{2 U}$ & -0.1761 & 0.4753 \\
Pyridine $\left(C_{2 V}\right)$ & $2 B_{2}$ & -0.6871 & -1.0246 \\
Pyrazine $\left(D_{2 h}\right)$ & $1 B_{2 U}$ & -0.1932 & 0.1178 \\
& $2 B_{2 U}$ & -0.6932 & -1.1521 \\
Pyrimidine $\left(C_{2 V}\right)$ & $3 B_{2}$ & -0.7826 & -0.8935 \\
Triplet cyclobutadiene ${ }^{\mathrm{a}}\left(D_{4 h}\right)$ & $1 E_{2 U}$ & -4.6154 & -5.4524 \\
& $2 E_{2 U}$ & -4.6154 & -5.4677 \\
& $3 E_{2 U}$ & -0.1886 & 0.4788 \\
Byphenylene $\left(D_{2 h}\right)$ & $4 E_{2 U}$ & -0.1886 & 0.4772 \\
& $2 B_{1 U}$ & -0.3867 & 0.0153 \\
& $4 B_{1 U}$ & -0.6803 & -2.3683 \\
Naphthalene $\left(D_{2 h}\right)$ & $5 B_{1 U}$ & -0.4949 & 0.5141 \\
& $2 B_{2 U}$ & -0.0323 & 0.0525 \\
& $3 B_{2 U}$ & -0.7520 & -1.5846 \\
& $5 B_{2 U}$ & -0.1742 & 0.3909 \\
\hline \hline
\end{tabular}

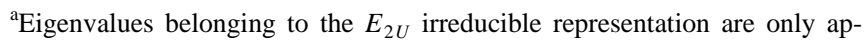
proximately degenerated because of small numerical inaccuracies.

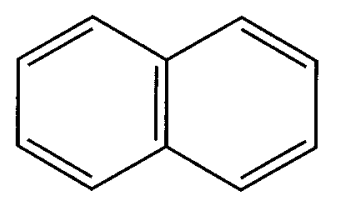

$D_{2}$ h 3(1) results achieved using hardnesses obtained from Eq. (5) are quite dependent on the basis set and the method of calculation. At the HF/aug-cc-pVTZ, HF/6-311G**, and the MP2/ 6-31G levels, the $1 B_{2 U^{\prime}}$ distortion violates the GMHP while the $2 B_{2 U^{\prime}}$ distortion follows it. With the B3LYP/6-31G method both $B_{2 U^{\prime}}$ displacements disobey the GMHP. Finally, using the HF and QCISD methods and the 6-31G basis set, it is found that the two $B_{2 U^{\prime}}$ distortions act in accordance with the GMHP. Given the variety of results obtained when using different methods and basis sets, it is clear that in order to determine the nuclear displacements that have a more marked GMHP or anti-GMHP one should diagonalize the Hessian matrix of the hardness with respect to the nontotally symmetric normal coordinates.

In line with the results obtained for the two original $B_{2 U}$ vibrational modes studied, we cannot attribute the breakdown of the GMHP and GMPP in these two $B_{2 U^{\prime}}$ distortions to the fact that the chemical potential and the external potential change significantly during these vibrations.

To sum up, we have shown that the diagonalization of the polarizability Hessian matrix allows us to easily separate GMPP and anti-GMPP vibrational distortions. The results obtained support the importance of the BLA distortions on the breakdown of the GMPP.

\section{Simple rules to determine whether a molecule will possess nontotally symmetric modes that disobey the GMPP.}

Let us now examine the series of molecules in Fig. 4 having in common that all of them possess nontotally symmetric BLA modes that break the GMPP. From this series, it would seem rational to conclude that any $\pi$-conjugated system having BLA vibrational movements would have nontotally symmetric modes that disobey the GMPP. Then, it would be reasonable to conclude that molecules in Fig. 3 such as cyclopentadiene or furan do not have nontotally symmetric modes that disobey the GMPP because these molecules do not possess BLA vibrational movements. However, it would not be possible to justify why molecules such as 
TABLE III. Static isotropic average polarizabilities, chemical potentials, and hardness for the molecular distortions of benzene along the two $B_{2 U^{\prime}} \mathrm{HF} / 6-31 \mathrm{G}$ postdiagonalization distortions depicted in Fig. 1. ${ }^{\mathrm{a}}$

\begin{tabular}{|c|c|c|c|c|c|c|c|}
\hline \multirow[b]{2}{*}{ Property } & & \multicolumn{3}{|c|}{$\mathrm{HF}$} & \multirow{2}{*}{$\begin{array}{c}\text { B3LYP } \\
6-31 G\end{array}$} & \multirow{2}{*}{$\begin{array}{c}\text { MP2 } \\
6-31 G\end{array}$} & \multirow{2}{*}{$\frac{\text { QCISD }}{6-31 G}$} \\
\hline & & $6-31 \mathrm{G}$ & $6-311 G^{* *}$ & aug-cc-pVTZ & & & \\
\hline \multirow[t]{3}{*}{$\alpha$} & Eq. & 51.09808 & 56.79026 & 66.74075 & 53.33033 & 52.98986 & 51.36533 \\
\hline & $1 B_{2 U^{\prime}} \pm 0.04^{\mathrm{b}}$ & 51.09181 & 56.78218 & 66.72941 & 53.32577 & 52.98244 & 51.35983 \\
\hline & $2 B_{2 U^{\prime}} \pm 0.04^{\mathrm{b}}$ & 51.10097 & 56.79370 & 66.74392 & 53.33337 & 52.99272 & 51.36804 \\
\hline \multirow[t]{3}{*}{$-2 \mu_{1}$} & Eq. & 0.185837 & 0.199504 & 0.308492 & 0.244452 & 0.188612 & 0.188872 \\
\hline & $1 B_{2 U^{\prime}} \pm 0.04^{\mathrm{b}}$ & 0.185838 & 0.199506 & 0.308539 & 0.244445 & 0.188612 & 0.188872 \\
\hline & $2 B_{2 U^{\prime}} \pm 0.04^{\mathrm{b}}$ & 0.185850 & 0.199516 & 0.308539 & 0.244463 & 0.188623 & 0.188883 \\
\hline \multirow[t]{3}{*}{$\eta_{1}$} & Eq. & 0.482066 & 0.472429 & 0.368479 & 0.251955 & 0.471510 & 0.471236 \\
\hline & $1 B_{2 U^{\prime}} \pm 0.04^{\mathrm{b}}$ & 0.482154 & 0.472527 & 0.368527 & 0.251999 & 0.471597 & 0.471322 \\
\hline & $2 B_{2 U^{\prime}} \pm 0.04^{\mathrm{b}}$ & 0.482071 & 0.472432 & 0.368527 & 0.251958 & 0.471514 & 0.471239 \\
\hline \multirow[t]{3}{*}{$-2 \mu_{2}$} & Eq. & 0.343657 & 0.357857 & 0.373143 & 0.248669 & 0.296006 & 0.325000 \\
\hline & $1 B_{2 U^{\prime}} \pm 0.04^{\mathrm{b}}$ & 0.345452 & 0.357862 & 0.373163 & 0.248664 & 0.312557 & 0.324896 \\
\hline & $2 B_{2 U^{\prime}} \pm 0.04^{\mathrm{b}}$ & 0.345552 & 0.359235 & 0.372144 & 0.248429 & 0.299513 & 0.325275 \\
\hline \multirow[t]{3}{*}{$\eta_{2}$} & Eq. & 0.576654 & 0.566325 & 0.549814 & 0.418974 & 0.532549 & 0.546491 \\
\hline & $1 B_{2 U^{\prime}} \pm 0.04^{\mathrm{b}}$ & 0.574638 & 0.566420 & 0.549900 & 0.419022 & 0.542280 & 0.546460 \\
\hline & $2 B_{2 U^{\prime}} \pm 0.04^{\mathrm{b}}$ & 0.574640 & 0.564768 & 0.547714 & 0.419014 & 0.529157 & 0.546136 \\
\hline
\end{tabular}

${ }^{\mathrm{a}} \alpha, \mu_{1}, \eta_{1}, \mu_{2}$, and $\eta_{2}$ are calculated using Eqs. (8), (6), (7), (4), and (5), respectively, all these values are given in a.u. $\mu_{1}$ and $\eta_{1}$ are calculated using the HOMO and LUMO energies (in hartrees) of the Hartree-Fock molecular orbitals, except for the B3LYP method for which the Kohn-Sham orbital energies were used.

${ }^{\mathrm{b}}$ The distortions are given in bohrs.

singlet cyclobutadiene, pyridazine, and cis and trans butadiene having BLA vibrational movements possess only nontotally symmetric modes that obey the GMPP. Even more surprising is the situation of such similar systems as the triplet cyclobutadiene $\left(D_{4 h}\right)$ and the singlet cyclobutadiene $\left(D_{2 h}\right)$, the first showing some nontotally symmetric modes that break the GMPP, and the second having only nontotally symmetric modes that fulfill the GMPP. The same is true for pyrazine $\left(D_{2 h}\right)$, pyrimidine $\left(C_{2 v}\right)$, and pyridazine $\left(C_{2 v}\right)$, which only differ in the relative positions of the two nitrogen atoms in the aromatic ring.

Figure 5 displays the schematic BLA distortion that is possible to draw just by looking at the geometry of the molecules cited in the previous paragraph. The BLA displacement of triplet cyclobutadiene transforms as the $E_{U}$ irreduc- ible representation, while for the singlet is possible to build two different BLA distortions, both belonging to the $A_{g}$ representation. If we repeat this process for pyrazine, pyrimidine, and pyridazine we find that the BLA movements belong to the $B_{2 U}, B_{2}$, and $A_{1}$ irreducible representations, respectively. Thus, only molecules having BLA vibrational displacements that do not transform as the totally symmetric representation of their symmetry group possess nontotally symmetric movements that disobey the generalized MPP. Remarkably, totally symmetric BLA vibrational motions such as those of singlet cyclobutadiene or pyridazine do not belong to the group of nontotally symmetric distortions that approximately meet the two conditions of Parr and Chattaraj. Therefore, the fact that the GMPP is not applicable in these cases is not surprising, simply because the polarizability is

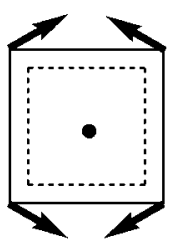

$\mathbf{D}_{4} \mathbf{h}$

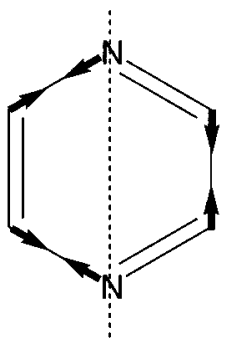

$\mathbf{D}_{\mathbf{2}} \mathbf{h}$

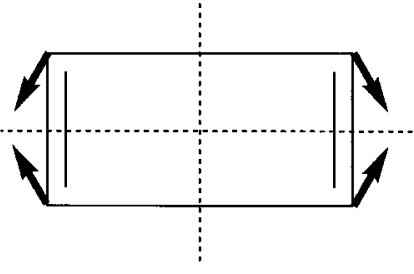

$\mathbf{D}_{2} \mathbf{h}$

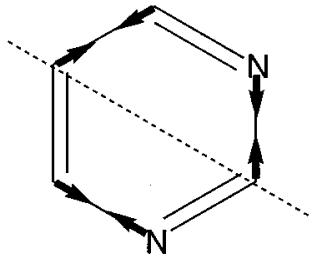

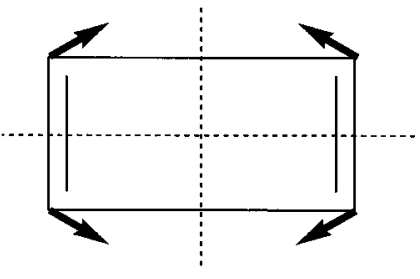

$\mathbf{D}_{\mathbf{2}} \mathbf{h}$

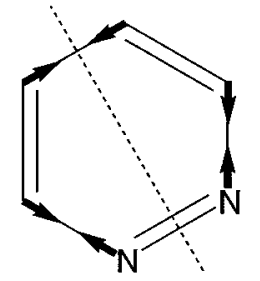

$\mathbf{C}_{2} \mathbf{v}$
FIG. 5. Different schematic BLA modes that can be drawn for triplet and singlet cyclobutadiene, pyrazine, pyrimidine, and pyridazine. 

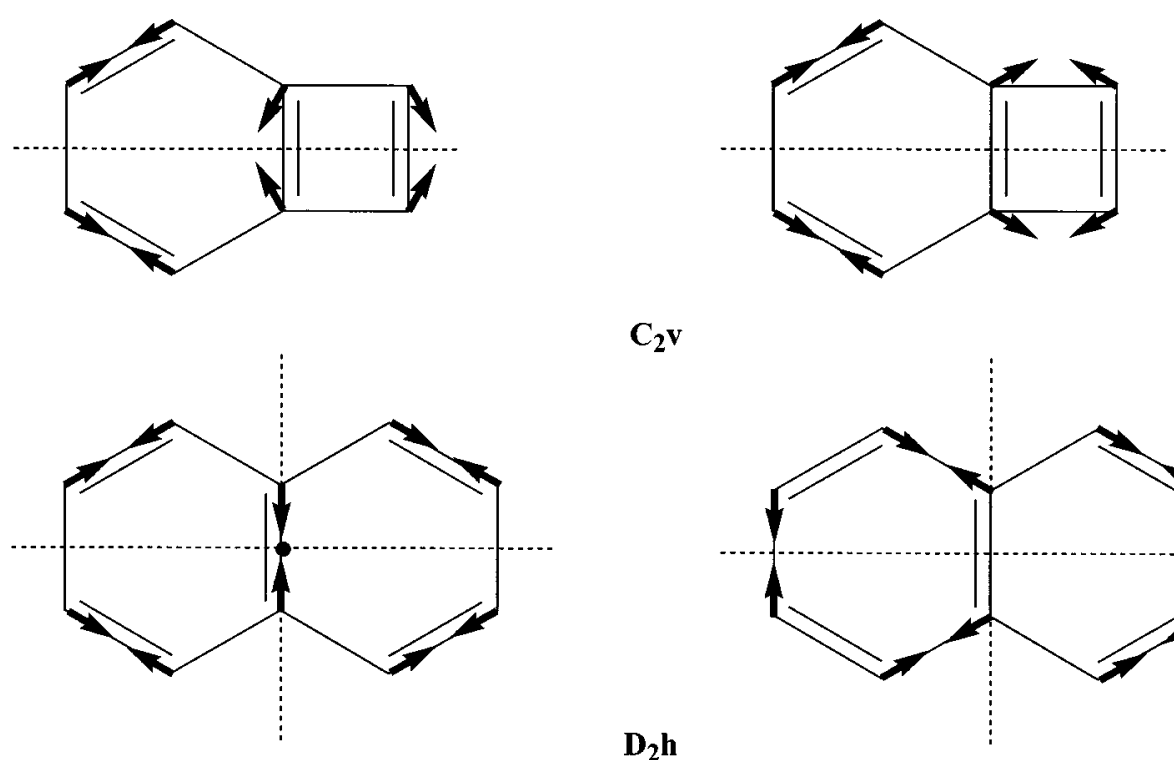

$\mathrm{C}_{2} \mathrm{v}$

$\mathbf{D}_{2} \mathbf{h}$

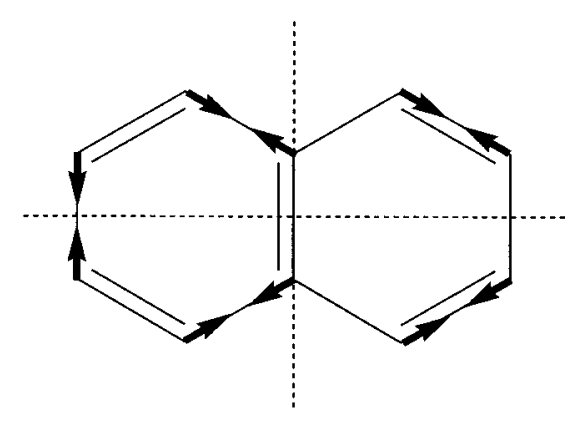

FIG. 6. Different schematic BLA modes that can be draw for benzocyclobutadiene and naphthalene. not an extremum with respect to distortions of the equilibrium structure along totally symmetric vibrational modes, as one can easily prove from symmetry considerations. ${ }^{15,17}$

When the molecules have different fused rings the analysis is slightly more complex, because in this case it is usually possible to draw different BLA distortions for the same molecule. For instance, Fig. 6 displays the two BLA movements of benzocyclobutadiene $\left(C_{2 V}\right)$ and naphthalene $\left(D_{2 h}\right)$, that one can depict just by looking at their geometries. The two BLA distortions in benzocyclobutadiene transform as the $A_{1}$ irreducible representation, while for naphthalene one BLA vibrational movement belongs to the $A_{g}$ representation and another transforms as the nontotally symmetric $B_{2 U}$ representation. As seen before, at variance with benzocyclobutadiene (Fig. 3), some nontotally symmetric vibrational modes of naphthalene (Fig. 4) have anti-GMPP character. Thus, we can conclude that if several BLA movements are possible, the molecules having one or more nontotally symmetric BLA movements will have nontotally symmetric vibrational modes that disobey the GMPP.

Using this information we have formulated the following set of simple rules that help us to a priori determine whether a given $\pi$-conjugated molecule will show nontotally symmetric vibrations that refuse to comply the GMPP:

Rule A: The molecule should have a BLA movement.

Rule B: Draw all possible BLA movements:

(a) If all BLA movements transform as the totally symmetric representation, the GMPP is obeyed by all nontotally symmetric modes of the studied system.

(b) If one or more BLA movements do not transform as the totally symmetric representation, the GMPP is disobeyed by some of the nontotally symmetric vibrational modes of the studied molecule.

Now, using these rules it is straightforward to check whether a given molecule has nontotally symmetric vibrational modes with anti-GMPP character. In particular, application of these rules to the set of molecules drawn in Figs. 3 and 4 affords the same qualitative results than the use of the diagonalization of the polarizability Hessian matrix methodology. Remarkably, not only it is possible to predict the existence but also the number of postdiagonalization nontotally symmetric distortions with anti-GMPP character. So, if one can drawn $n$ nontotally symmetric BLA distortions in a molecule, then this molecule will possess $n$ nontotally symmetric distortions that disobey the GMPP, as one can see comparing the number of negative eigenvalues of the A matrix gathered in Table II and the BLA distortions depicted in Figs. 5 and 6.

Let us finish the discussion with two comments. First, despite these rules have been derived and proven for the GMPP only, we have found that all molecules that violate the GMPP, also break the GMHP when the hardness is calculated using the HOMO and LUMO energies [Eq. (7)]. ${ }^{49} \mathrm{We}$ have also noticed that, in general, the modes that violate the GMPP, more manifestly break the GMHP using hardness obtained from Eq. (7). Second, we consider that the rules proposed here have a general applicability for $\pi$-conjugated systems. However, in the case of large molecules, with a substantial number of different modes, the possibility that a certain combination of vibrational modes may break the GMPP or GMHP, despite all BLA modes of this molecule belonging to the totally symmetric representation, cannot be rule out. Research in this direction is currently under way in our laboratory.

\section{CONCLUSIONS}

We have confirmed in this work that not only in chemical reactions but also even in the favorable case of nontotally symmetric vibrations where the chemical and external potentials keep approximately constant, the GMHP and GMPP may not be obeyed. This is an important result since it is relevant to establish the conditions at which the generalized versions of the MHP and MPP hold. We have also devised a method that allows an accurate determination of the nontotally symmetric molecular distortions with more marked GMPP or anti-GMPP character through diagonalization of 
the polarizability Hessian matrix. The results obtained with this method are basis set and method independent. Finally, we have derived a set of simple rules that for a given $\pi$-conjugated molecule allows to a priori predict the existence and the number of nontotally symmetric anti-GMPP distortions without the need to perform calculations. With respect to the GMHP, the results strongly depend on the scheme used to calculate the hardness, but when the Koopmans' approximation is used the same rules are valid to predict the existence of vibrational modes that disobey the GMHP.

\section{ACKNOWLEDGMENTS}

Support for this work under Grants Nos. BQU200204112-C02-02 and PB98-0457-C02-01 from the Dirección General de Enseñanza Superior e Investigación Científica y Técnica (MEC-Spain) is acknowledged. One of the authors (M.T.) thanks the Generalitat of Catalunya for financial help through CIRIT Project No. FI/01-00699. We are grateful to Professors Juvencio Robles and Alejandro Toro-Labbé for helpful comments. M.S. thanks the DURSI (Generalitat de Catalunya) for financial support through the Distinguished University Research Promotion, 2001.

${ }^{1}$ M. Solà An. R. Soc. Esp. Fis. Quim. 97, 12 (2001).

${ }^{2}$ P. W. Atkins, The Second Law (Scientific American, New York, 1994).

${ }^{3}$ G. S. Hammond, J. Am. Chem. Soc. 77, 334 (1955).

${ }^{4}$ A. Pross, Theoretical \& Physical Principles of Organic Reactivity (Wiley, New York, 1995).

${ }^{5}$ R. B. Woodward and R. Hoffmann, J. Am. Chem. Soc. 87, 395 (1965); R. B. Woodward and R. Hoffmann, The Conservation of Orbital Symmetry (Verlag Chemie, Weinheim, 1970); R. B. Woodward and R. Hoffmann, Angew. Chem. Int. Ed. Engl. 8, 781 (1969); Acc. Chem. Res. 1, 17 (1968).

${ }^{6}$ P. K. Chattaraj, S. Nath, and A. B. Sannigrahi, Chem. Phys. Lett. 212, 223 (1993); J. Phys. Chem. 98, 9143 (1994).

${ }^{7}$ R. T. Sanderson, Science 121, 207 (1955); R. T. Sanderson, Chemical Bonds and Bond Energy (Academic, New York, 1976).

${ }^{8}$ R. G. Parr and R. G. Pearson, J. Am. Chem. Soc. 105, 7512 (1983).

${ }^{9}$ R. G. Pearson, Chemical Hardness: Applications from Molecules to Solids (Wiley-VCH, Oxford, 1997)

${ }^{10}$ R. G. Pearson, J. Chem. Educ. 64, 561 (1987); 76, 267 (1999).

${ }^{11}$ R. G. Parr and P. K. Chattaraj, J. Am. Chem. Soc. 113, 1854 (1991).

${ }^{12}$ T. K. Ghanty and S. K. Ghosh, J. Phys. Chem. 100, 12295 (1996).

${ }^{13}$ P. K. Chattaraj and S. Sengupta, J. Phys. Chem. 100, 16126 (1996).

${ }^{14}$ T. K. Ghanty and S. K. Ghosh, J. Phys. Chem. 97, 4951 (1993).

${ }^{15}$ R. G. Pearson and W. E. Palke, J. Phys. Chem. 96, 3283 (1992)

${ }^{16}$ S. Pal, N. Vaval, and R. Roy, J. Phys. Chem. 97, 4404 (1993).

${ }^{17}$ G. Makov, J. Phys. Chem. 99, 9337 (1995).

${ }^{18}$ P. K. Chattaraj, P. Fuentealba, P. Jaque, and A. Toro-Labbé, J. Phys. Chem. A 103, 9307 (1999).

${ }^{19}$ G. I. Cárdenas-Jirón, J. Lahsen, and A. Toro-Labbé, J. Phys. Chem. 99, 5325 (1995); G. I. Cárdenas-Jirón and A. Toro-Labbé, ibid. 99, 12730 (1995); G. I. Cárdenas-Jirón, S. Gutiérrez-Oliva, J. Melin, and A. ToroLabbé, J. Phys. Chem. A 101, 4621 (1997); G. I. Cárdenas-Jirón and A. Toro-Labbé, J. Mol. Struct.: THEOCHEM 390, 79 (1997); S. Gutiérrez-
Oliva, J. R. Letelier, and A. Toro-Labbé, Mol. Phys. 96, 61 (1999).

${ }^{20}$ P. K. Chattaraj and A. Poddar, J. Phys. Chem. A 103, 1274 (1999).

${ }^{21}$ P. Fuentealba, Y. Simón-Manso, and P. K. Chattaraj, J. Phys. Chem. A 104, 3185 (2000).

${ }^{22}$ Z. Zhou and R. G. Parr, J. Am. Chem. Soc. 111, 7371 (1989).

${ }^{23}$ D. Datta, J. Phys. Chem. 96, 2409 (1992).

${ }^{24}$ T. Kar and S. Scheiner, J. Phys. Chem. 99, 8121 (1995).

${ }^{25}$ P. K. Chattaraj, A. Cedillo, R. G. Parr, and E. M. Arnett, J. Org. Chem. 60, 4707 (1995).

${ }^{26}$ T. Kar, S. Scheiner, and A. B. Sannigrahi, J. Phys. Chem. A 102, 5967 (1998).

${ }^{27}$ T. Mineva, E. Sicilia, and N. Russo, J. Am. Chem. Soc. 120, 9053 (1998).

${ }^{28}$ A. Toro-Labbé, J. Phys. Chem. A 103, 4398 (1999).

${ }^{29}$ M. Solà and A. Toro-Labbé, J. Phys. Chem. A 103, 8847 (1999).

${ }^{30}$ P. Jaque and A. Toro-Labbé, J. Phys. Chem. A 104, 995 (2000).

${ }^{31}$ P. K. Chattaraj, P. Fuentealba, B. Gómez, and R. Contreras, J. Am. Chem. Soc. 122, 348 (2000).

${ }^{32}$ U. Hohm, J. Phys. Chem. A 104, 8418 (2000).

${ }^{33}$ E. Sicilia, N. Russo, and T. Mineva, J. Phys. Chem. A 105, 442 (2001).

${ }^{34}$ L. Salem, Electrons in Chemical Reactions (Wiley, New York, 1982), Chap. 2.

${ }^{35}$ M. J. Colthurst and A. Williams, J. Chem. Soc.: Perkin Trans. 2, 1493 (1997); S. S. Kim, H. R. Kim, H. B. Kim, S. J. Youn, and C. J. Kim, J. Am. Chem. Soc. 116, 2754 (1994).

${ }^{36}$ M. Solà, J. Mestres, R. Carbó, and M. Duran, J. Am. Chem. Soc. 116, 5909 (1994)

${ }^{37}$ By definition, $v_{\text {en }}=\int \rho(\mathbf{r}) v(\mathbf{r}) d \mathbf{r}$, and therefore if $v_{\text {en }}$ keeps constant for a particular distortion of the molecular structure, one may assume that $v(\mathbf{r})$ also remains roughly constant during this distortion.

${ }^{38}$ M. Torrent-Sucarrat, J. M. Luis, M. Duran, and M. Solà, J. Am. Chem. Soc. 123, 7951 (2001).

${ }^{39}$ A. D. Becke, J. Chem. Phys. 98, 5648 (1993); C. Lee, W. Yang, and R. G. Parr, Phys. Rev. B 37, 785 (1988); P. J. Stevens, F. J. Devlin, C. F. Chablowski, and M. J. Frisch, J. Phys. Chem. 98, 11623 (1994).

${ }^{40}$ C. Møller and M. S. Plesset, Phys. Rev. 46, 618 (1934).

${ }^{41}$ J. A. Pople, M. Head-Gordon, and K. Raghavachari, J. Chem. Phys. 87, 5968 (1987)

${ }^{42}$ W. J. Hehre, L. Radom, P. v. R. Schleyer, and J. A. Pople, Ab Initio Molecular Orbital Theory (Wiley, New York, 1986).

${ }^{43}$ R. A. Kendall, T. H. Dunning, Jr. and R. J. Harrison, J. Chem. Phys. 96, 6796 (1992)

${ }^{44}$ M. J. Frisch, G. W. Trucks, H. B. Schlegel et al., Gaussian 98, Revision A.10, Gaussian, Inc., Pittsburgh, PA, 1998.

${ }^{45}$ R. G. Parr and W. Yang, Density-Functional Theory of Atoms and Molecules (Oxford University Press, New York, 1989).

${ }^{46}$ R. G. Pearson, J. Am. Chem. Soc. 107, 6801 (1985).

${ }^{47}$ See EPAPS Document No. E-JCPSA6-117-313245 for tables containing the 6-31G and 6-311G** HF, B3LYP, and MP2 results and the QCISD/6$31 \mathrm{G}$ values of the chemical potential, hardness, and polarizability for all molecular distortions of benzene along its nontotally symmetric normal modes. A direct link to this document may be found in the online article's HTML reference section. The document may also be reached via the EPAPS homepage (http://www.aip.org/pubservs/epaps.html) or from ftp.aip.org in the directory /epaps/. See the EPAPS homepage for more information.

${ }^{48}$ (a) F. De Proft and P. Geerlings, J. Chem. Phys. 106, 3270 (1997); (b) M. Torrent-Sucarrat, M. Duran, and M. Solà, J. Phys. Chem. A 106, 4632 (2002).

${ }^{49}$ We have not attempted to calculate the hardness through Eq. (5) for systems depicted in Figs. 3 and 4 because of computational limitations. 